\title{
Body Position Adjustment May Facilitate Capsule Endoscopic Real-Time Examination in Patients with a Large Amount of Food Retention in the Stomach
}

\author{
Ju-Hua Gou and Bing-Qiang Zhang \\ Department of Gastroenterology, First Affiliated Hospital of Chongqing Medical University, Chongqing, China
}

\begin{abstract}
A patient with acute obscure gastrointestinal bleeding was found to have a large amount of food retention in the stomach after fasting for $>12$ hours. We tried to adjust the patient's body position to facilitate capsule endoscopic examination. The patient laid on the bed on his right side, which is the position required for a normal procedure, and then his hip was raised while his upper body was lowered gradually until the pylorus appeared at the center of the screen of the real-time monitor. It took 15 minutes of body position adjustment to make the pylorus appear at the center of the monitor and another 5 minutes for the capsule endoscope to enter the duodenum. The lesion was ultimately found at the terminal small intestine.
\end{abstract}

Key Words: Capsule endoscopy; Body position; Real-time monitoring

\section{INTRODUCTION}

Capsule endoscopic examination is a valuable method for finding gastrointestinal (GI) lesions such as a tumor, and those causing bleeding and infection. It causes minimal harm and is easily accepted by patients. ${ }^{1,2}$ However, bowel preparation can be limited and dangerous if the patients have active upper GI bleeding. Even fasting for $>12$ hours may not be adequate to empty the gastric food deposit, especially in older patients or those with diabetes patients. Therefore, a large amount of food retention in the stomach hinders the success rate of capsule endoscopic examination. Thus, it remains a practical clinical challenge to overcome such obstacles to improve the accuracy and effectiveness of capsule endoscopy. Here, we report a case study in which body position adjustment facilitated capsule endoscopic examination of a patient with significant

Received: October 12, 2013 Revised: November 30, 2013

Accepted: December 21, 2013

Correspondence: Bing-Qiang Zhang

Department of Gastroenterology, First Affiliated Hospital of Chongqing Medical University, No. 1, Youyi Road, Yuan Jia Gang, Yuzhong, Chongqing 400016, China

Tel: +86-23-8901-2962, Fax: +86-23-8901-2962

E-mail: zhbingqiang@163.com

(c) This is an Open Access article distributed under the terms of the Creative Commons Attribution Non-Commercial License (http://creativecommons.org/ licenses/by-nc/3.0) which permits unrestricted non-commercial use, distribution, and reproduction in any medium, provided the original work is properly cited. food retention in the stomach.

\section{CASE REPORT}

A 54-year-old male patient presented with GI bleeding of unknown origin that had started 2 weeks previously. He had approximately 500 to $1,000 \mathrm{~mL} /$ day, fresh red or dark red blood in the stool. He had no nausea and vomiting, with negative findings on upper gastric endoscopy and colonoscopy. He experienced bleeding recurrence 2 hours before saw a doctor, and was emergently admitted to the hospital. He had approximately 500 to $1,000 \mathrm{~mL} /$ day of fresh red/dark red blood in the stool. Blood transfusion and somatostatin were administered without considerable improvement. A stool test showed three to five red blood cells per high-magnification field. His hemoglobin level was $93 \mathrm{~g} / \mathrm{L}$. Computed tomography (CT) and CT angiography, including CT ateriography and CT venography, failed to yield a definitive diagnosis. He was prepared for capsule endoscopy by fasting for an additional 12 hours. He was asked to swallow the capsule endoscope with a small cup of cool water, and real-time monitoring of the procedure was set up with a laptop computer beside his bed. The capsule endoscope used was of the model OMOM (Chongqing Jinshan Science and Technology Co., Ltd., Chongqing, China) with the following features:size, $13 \times 27.9 \mathrm{~mm}$; weight, 

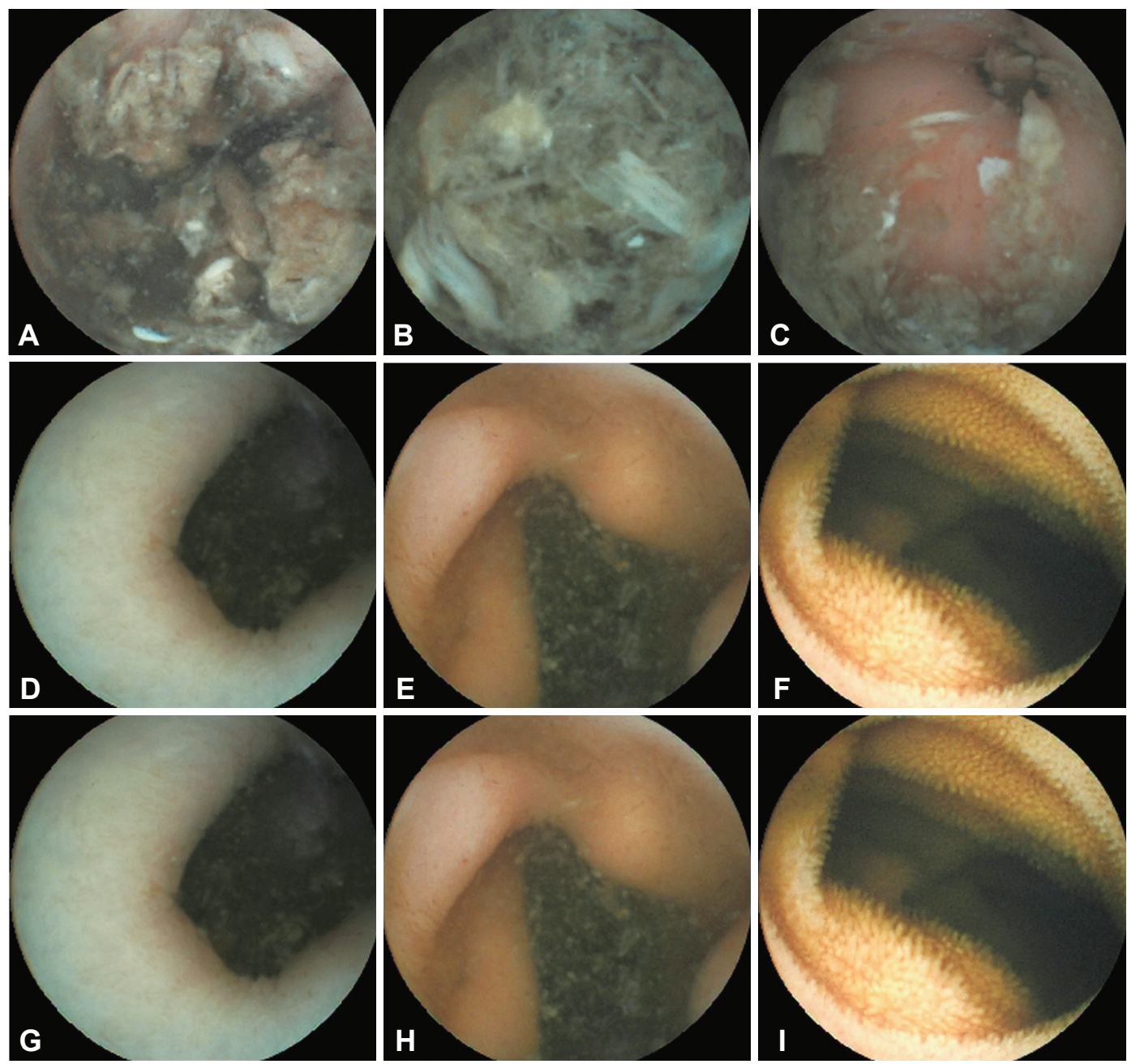

Fig. 1. Serial images of the capsule endoscope passing from the stomach to the colon. (A, B) They show the presence of too much food in the stomach. (C) It shows the appearance of the pylorus on the monitor screen owing to the change in body position. (D) It shows the pylorus positioned at the center of the screen. (E) It shows the capsule endoscope entering the duodenum. (F) It shows the capsule endoscope entering the jejunum. (G) It shows an ulcer lesion in the terminal ileum. $(\mathrm{H})$ It shows fresh blood in the cecum. (I) It shows the capsule endoscope entering the colon.

approximately $6 \mathrm{~g}$; one side view; view angle, $140 \pm 10^{\circ}$; sample rate, 2 frames per second $\pm 10 \%$; and performance time, approximately 7 to 9 hours. At the start of capsule endoscopy, we found significant food retention in the patient's stomach (Fig. 1A, B). The examination quality did not improve even after the patient was instructed to drink some water. To avoid possibly worsening of bleeding, we did not administer any prokinetic drugs. Instead, the patient was asked to lie on the bed on his right side, as is required in normal procedures, followed by raising his hip gradually and lowering his head (Fig. 2). Meanwhile, the real-time monitoring system of the capsule endoscope was adjusted until the pylorus was positioned at the center of the screen. We found that it took 15 minutes of body position adjustment to make the pylorus appear at the center of viewer (Fig. 1C, D) and another 5 minutes for the capsule endoscope to enter the duodenum (Fig. $1 \mathrm{E})$, then jejunum. After 2 hours, the capsule endoscope en- tered the cecum (Fig. 1F) and ulcer lesions were seen at the terminal ileum (Fig. 1G) with fresh blood in the cecum (Fig. $1 \mathrm{H})$, and there was no fresh blood in another part of colon (Fig. 1I). The whole process was recorded on Supplementary Video 1 (available online at http://www.e-ce.org/).

\section{DISCUSSION}

The classic right side-lying position has been commonly used for capsule endoscopy in clinical practice and often works well. The mean time taken to enter the pylorus is 30 minutes to 1 hour. However, when there is significant food retention in the stomach, the right side-lying position method does not work well. We reviewed some capsule endoscopy procedures and found that in most procedures performed in the right side-lying position, did not succeed in making the pylorus appear at the center of the screen. Thus, we tried to 


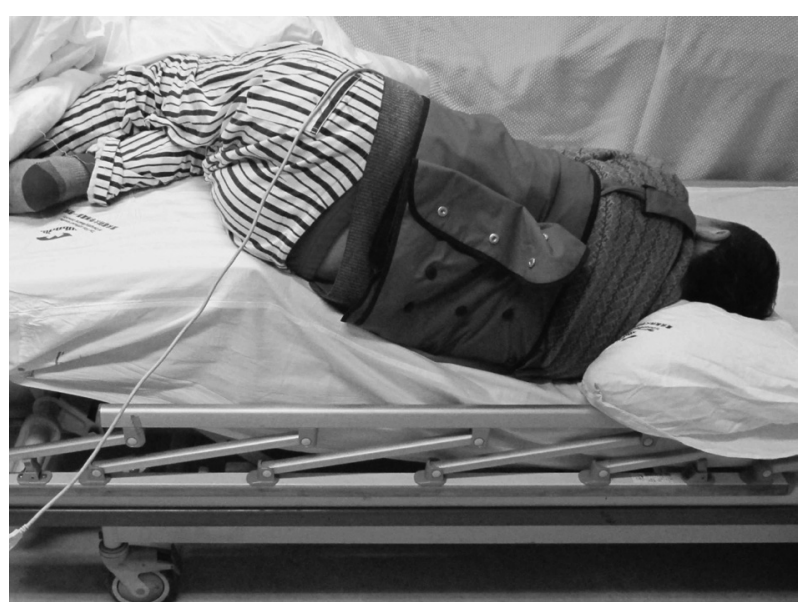

Fig. 2. Adapting a special body position to allow the pylorus to appear at the center of the screen.
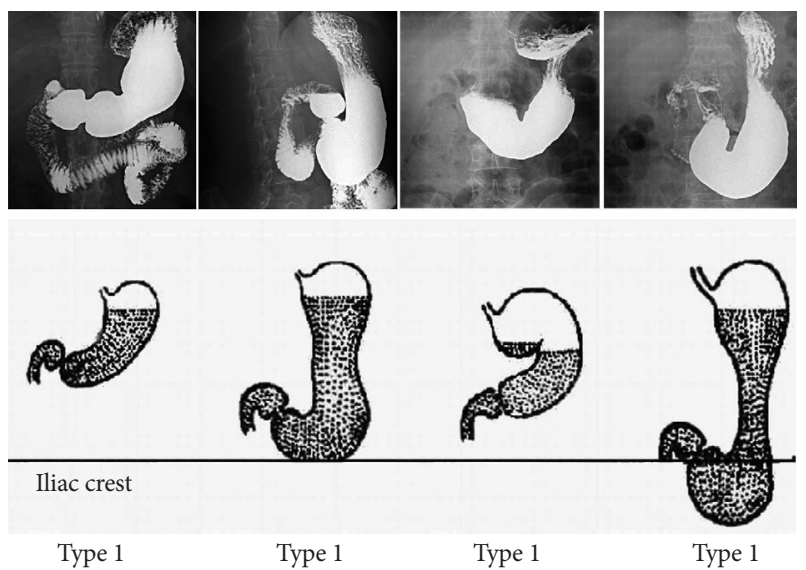

Fig. 3. Gastric profiles and locations of the capsule endoscope in the pylorus. The classic stomach types on barium meal radiographic examination, type 1 to type 4; the different gastric profiles would influence the location of capsule endoscope in the pylorus when the patient assumes the right lateral position.

elevate the hip and lower the head, and found that this maneuver helped position the pylorus at the center of the viewer quickly, and the capsule endoscope easily entered the duodenum, similar to when performing a gastric endoscopy procedure.

We also reviewed the radiography results of barium meal examination (Fig. 3) in the four classic stomach types, and found that only type 1 is suitable for the right side-lying position. However, if the body position is adjusted, all four stomach types would be suitable for the right side-lying position (Fig. 3).

Höög et al. ${ }^{3}$ analyzed 2,300 patients who underwent capsule endoscopy and found that the rate of incomplete examinations was $20 \%$. The risk factors include older age, male sex, and Crohn disease. Slow gastric transit with the capsule in the stomach for the entire recording time was found in $14 \%$ of incomplete examination cases and was a major contributing factor for lowering the success rate. The use of prokinetics has not improved the gastric transit time. Conversely, approximately $1.3 \%$ of capsule retention cases occurred in patients with Crohn disease and/or GI tumors.

A real-time viewer is frequently used for manually placing the capsule in the duodenum, and in this case, gastric retention may improve the possibility of complete transit. Bass and Misiewicz ${ }^{1}$ used a real-time viewer to determine the delay in capsule transit, and used an endoscope to pass the capsule endoscope into the duodenum. However, in our case, the patient had significant food retention in the stomach, and thus this method would not be useful. Shiotani et al. ${ }^{4}$ used an external real-time image viewer and found that it facilitated completion of capsule endoscopy. To improve the delay, water or intravenous metoclopramide was administered. In our case, the patient was instructed to drink some water, but it failed to work. Intravenous metoclopramide would increase bowel movement but may worsen the bleeding; therefore, we chose not to administer this drug.

Obscure GI bleeding (OGIB) is the most common indication for capsule endoscopy as outlined in the 2013 Guideline for Capsule Endoscopy; ${ }^{5}$ for a moderate evidence grade, capsule endoscopy is strongly suggested to be performed as soon as possible for OGIB. The diagnostic rate of capsule endoscopy for OGIB varied between $50 \%$ and $60 \%{ }^{6-8}$ It is conceivable that using magnetic guidance and a real-time viewer may help the capsule endoscope enter the pylorus more easily and quickly. The capsule endoscopy system includes externally rechargeable batteries (by using radiofrequency, microwave, ultrasound, or electric induction) or may even be batteryfree, which will find more useful data aiding diagnosis. ${ }^{9,10}$

\section{Conflicts of Interest}

The authors have no financial conflicts of interest.

\section{Acknowledgments}

We thank Dr. T.C. He of The University of Chicago Medical Center for critically reading and commenting on the manuscript.

\section{REFERENCES}

1. Bass LM, Misiewicz L. Use of a real-time viewer for endoscopic deployment of capsule endoscope in the pediatric population. J Pediatr Gastroenterol Nutr 2012;55:552-555.

2. Orlando G, Luppino IM, Lerose MA, et al. Feasibility of capsule endoscopy in elderly patients with obscure gastrointestinal bleeding. An upto-date report. BMC Surg 2012;12 Suppl 1:S30.

3. Höög CM, Bark LÅ, Arkani J, Gorsetman J, Broström O, Sjöqvist U. Capsule retentions and incomplete capsule endoscopy examinations: an analysis of 2300 examinations. Gastroenterol Res Pract 2012;2012: 518718 .

4. Shiotani A, Honda K, Kawakami M, et al. Use of an external real-time image viewer coupled with prespecified actions enhanced the complete examinations for capsule endoscopy. J Gastroenterol Hepatol 2011;26:1270-1274.

5. Shim KN, Moon JS, Chang DK, et al. Guideline for capsule endoscopy: obscure gastrointestinal bleeding. Clin Endosc 2013;46:45-53. 
6. Eliakim R. Video capsule endoscopy of the small bowel. Curr Opin Gastroenterol 2008;24:159-163.

7. Nakamura T, Terano A. Capsule endoscopy: past, present, and future. J Gastroenterol 2008;43:93-99.

8. Lecleire S, Iwanicki-Caron I, Di-Fiore A, et al. Yield and impact of emer- gency capsule enteroscopy in severe obscure-overt gastrointestinal bleeding. Endoscopy 2012;44:337-342.

9. Rey JF. The future of capsule endoscopy. Keio J Med 2013;62:41-46.

10. Neumann H, Fry LC, Neurath MF. Review article on current applications and future concepts of capsule endoscopy. Digestion 2013;87:91-99. 